\title{
Automatic Histogram Threshold using Fuzzy Measures
}

\author{
Nuno Vieira Lopes, Pedro Couto, Humberto Bustince, Member, IEEE, and Pedro Melo-Pinto
}

\begin{abstract}
In this paper, an automatic histogram threshold approach based on a fuzziness measure is presented. This work is an improvement of an existing method. Using fuzzy logic concepts, the problems involved in finding the minimum of a criterion function are avoided. Similarity between gray levels is the key to find an optimal threshold. Two initial regions of gray levels, located at the boundaries of the histogram, are defined. Then, using an index of fuzziness, a similarity process is started to find the threshold point. A significant contrast between objects and background is assumed. Previous histogram equalization is used in small contrast images. No prior knowledge of the image is required.
\end{abstract}

\section{INTRODUCTION}

I MAGE segmentation plays an important role in computer vision and image processing applications. Segmentation of nontrivial images is one of the most difficult tasks in image processing. Segmentation accuracy determines the eventual success or failure of computerized analysis procedures. Segmentation of an image entails the division or separation of the image into regions of similar attribute. For a monochrome image, the most basic attribute for segmentation is image luminance amplitude [1].

Segmentation based on gray level histogram thresholding is a method to divide an image containing two regions of interest: object and background. In fact, applying this threshold to the whole image, pixels whose gray level is under this value are assigned to a region and the remainder to the other. Histograms of images with two distinct regions are formed by two peaks separated by a deep valley called bimodal histograms. In such cases, the threshold value must be located on the valley region. When the image histogram does not exhibit a clear separation, ordinary thresholding techniques might perform poorly. Fuzzy set theory provides a new tool to deal with multimodal histograms. It can incorporate human perception and linguistic concepts such as similarity, and has been successfully applied to image thresholding [2]-[9].

The remainder of this paper is organized as follows. In Section II a background review on thresholding methods is

N. Vieira Lopes is with the Department of Electrical Engineering, Escola Superior de Tecnologia e Gestão - IPL, Campus 1, Apartado 4045, 2411-901 Leiria, Portugal and CITAB - Centro de Investigação e de Tecnologias Agro-Ambientais e Biológicas, Universidade de Trás-os-Montes e Alto Douro, Quinta de Prados, 5000-911 Vila Real, Portugal e-mail: nlopes@estg.ipleiria.pt.

H. Bustince is with the Departamento de Automática y Computación, Universidad Pública de Navarra, Campus Arrosadia s/n, 31006, Pamplona, Spain e-mail: bustince@unavarra.es.

P. Melo-Pinto and P. Couto are with CITAB - Centro de Investigação e de Tecnologias Agro-Ambientais e Biológicas, Universidade de Trás-osMontes e Alto Douro, Quinta de Prados, 5000-911 Vila Real, Portugal e-mail: pmelo@utad.pt; pcouto@utad.pt. presented. A general description of the fuzzy set theory and index of fuzziness measuring is presented in Section III. The existing method is described in Section IV. The proposed method is presented in Section V. Limitations and detected problems of the existing method are also discussed. Section VI shows comparative results to illustrate the effectiveness of the proposed approach and Section VII presents the final conclusions.

\section{THRESHOLDING ALGORITHMS}

In general, threshold selection can be categorized into two classes, local and global methods. Using global thresholding methods an entire image is binarized with a single threshold, while the local methods divide the given image into a number of sub-images and select a suitable threshold for each subimage. The global thresholding techniques are easy to implement and computationally less demanding, therefore they are more suitable than local methods in terms of many real image processing applications. Many different approaches are used in image thresholding.

Rosenfeld's convex hull method is based on analyzing the concavity structure of the histogram defined by its convex hull [10]. When the convex hull of the histogram is calculated, the deepest concavity points become candidates for the threshold value. A variation of this method can be found in [11].

Ridler and Calvard algorithm [12] uses an iterative technique for choosing a threshold value. At iteration $n$, a new threshold $T_{n}$ is established using the average of the foreground and background class means. The process is repeated until the changes in $T_{n}$ become sufficiently small.

Otsu's technique [13] is based on discrimination analysis, in which the optimal threshold value calculation is based on the minimization of the weighted sum of the object and background pixels within-class variances.

In Kittler and Illingworth's minimum error thresholding method it is assumed that the image can be characterized by a mixture distribution of object and background pixels [14].

Jawahar et al. [6] propose a fuzzy thresholding scheme based on Fuzzy C-means clustering. The problem of fuzzy clustering is that of partitioning the set of $n$ sample points into $c$ classes. The algorithm is an iterative optimization that minimizes one cost function. Two extensions of this algorithm are found in [7] and [3].

Kapur et al. [15] propose a method based on the previous work of Pun [16] that first applied the concept of entropy to thresholding. This method interprets the image object and background as two different information sources. When the 
sum of the object and background entropies reaches its maximum, the image is said to be optimally thresholded.

Huang and Wang [8] assign the memberships to the pixel with the help of the relationship between its gray value and mean gray value of the region to which it belongs. In this case, the image is regarded as a single fuzzy set where the membership distribution reflects the compatibility of the pixels to the region to which it belongs.

An exhaustive survey of image thresholding methods can be found in [17].

\section{General Definitions}

\section{A. Fuzzy Set Theory}

Fuzzy set theory assigns a membership degree to all elements among the universe of discourse according to their potential to fit in some class. The membership degree can be expressed by a mathematical function $\mu_{A}\left(x_{i}\right)$ that assigns, to each element in the set, a membership degree between 0 and 1. Let $X$ be the universe (finite and not empty) of discourse and $x_{i}$ an element of $X$. A fuzzy set $A$ in $X$ is defined as

$$
A=\left\{\left(x_{i}, \mu_{A}\left(x_{i}\right)\right) \mid x_{i} \in X\right\}
$$

The S-function is used for modeling the membership degrees [18]. This type of function is suitable to represent the set of bright pixels and is defined as

$$
\begin{aligned}
\mu_{A S}(x)=S(x ; a, b, c) \\
= \begin{cases}0, & x \leq a \\
2\{(x-a) /(c-a)\}^{2}, & a \leq x \leq b \\
1-2\{(x-c) /(c-a)\}^{2}, & b \leq x \leq c \\
1, & x \geq c .\end{cases}
\end{aligned}
$$

where $b=\frac{1}{2}(a+c)$. The $\mathrm{S}$-function can be controlled through parameters $a$ and $c$. Parameter $b$ is called the crossover point where $\mu_{A S}(b)=0.5$. The higher the gray level of a pixel (closer to white), the higher membership value and vice versa. A typical shape of the S-function is presented in Fig. 1. The $\mathrm{Z}$-function is used to represent the dark pixels and is defined by an expression obtained from S-function as follows

$$
\mu_{A Z}(x)=Z(x ; a, b, c)=1-S(x ; a, b, c)
$$

Both membership functions could be seen, simultaneously, in Fig. 2. The S-function in the right side of the histogram and the Z-function in the left.

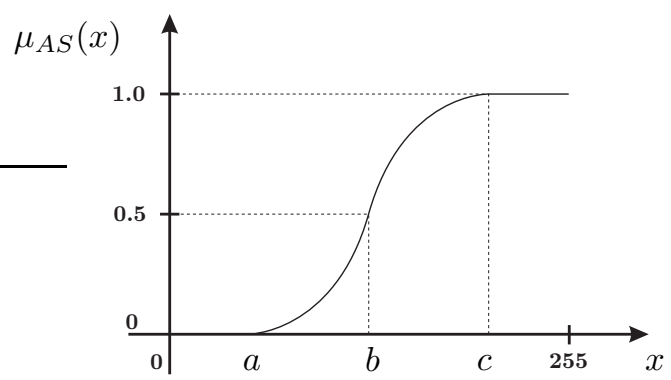

Fig. 1. Typical shape of the S-function

\section{B. Measures of fuzziness}

A reasonable approach to estimate the average ambiguity in fuzzy sets is measuring its fuzziness [19]. The fuzziness of a crisp set should be zero, as there is no ambiguity about whether an element belongs to the set or not. If $\mu_{A}(x)=0.5, \forall x$, the set is maximally ambiguous and its fuzziness should be maximum. Degrees of membership near 0 or 1 indicate lower fuzziness, as the ambiguity decreases. Kaufmann in [20] introduced an index of fuzziness (IF) comparing a fuzzy set with its nearest crisp set. A fuzzy set $A^{*}$ is called crisp set of $A$ if the following conditions are satisfied

$$
\mu_{A^{*}}(x)= \begin{cases}0, & \text { if } \mu_{A}(x)<0.5 \\ 1, & \text { if } \mu_{A}(x) \geq 0.5\end{cases}
$$

This index is calculated by measuring the normalized distance between $A$ and $A^{*}$ defined as

$$
\psi_{k}(A)=\frac{2}{n^{1 / k}}\left[\sum_{i=1}^{n}\left|\mu_{A}\left(x_{i}\right)-\mu_{A^{*}}\left(x_{i}\right)\right|^{k}\right]^{1 / k}
$$

where $n$ is the number of elements in $A, k \in[1, \infty[$. Depending if $k=1$ or 2 , the index of fuzziness is called linear or quadratic. Such an index reflects the ambiguity in a set of elements. If a fuzzy set shows low index of fuzziness there exists a low ambiguity among elements.

\section{Existing MEthoD}

This work is an improvement of an existing method based on a fuzziness measure to find the threshold value in a gray image histogram [4], [5]. The method incorporates fuzzy concepts that are more able to deal with object edges and ambiguity and avoids the problems involved in finding the minimum of a function. However it has some limitations concerning the initialization of the seed subsets. To achieve an automatic process these limitations must be overcome. In order to implement the thresholding algorithm on a basis of the concept of similarity between gray levels, Tobias \& Seara made the assumptions that there exists a significant contrast between the objects and background and that the gray level is the universe of discourse, a one-dimensional set, denoted by $X$. The purpose is to split the image histogram into two crisp subsets, object subset $O$ and background subset $F$, using the measure of fuzziness previously defined. The initial fuzzy subsets, denoted by $B$ and $W$, are associated with initial histogram intervals located at the beginning and the end regions of the histogram. The gray levels in each of these initial intervals have the intuitive property of belonging with certainty to the final subsets object or background. For dark objects $B \subset O$ and $W \subset F$, for light objects $B \subset F$ and $W \subset O$. These initial fuzzy subsets, $W$ and $B$, are modeled by the $S$ and $Z$ membership functions, respectively. The parameters of the $S$ and $Z$ functions are variable to adjust its shape as a function of the set of elements [5].

These subsets are a seed for starting the similarity measure process. A fuzzy region placed between these initial intervals is defined as depicted in Fig. 2. Then, to obtain the segmented version of the gray level image, we have to classify each gray level of the fuzzy region as being object or background. The 
classification procedure is done by adding to each of the seed subsets a gray level $x_{i}$ picked from the fuzzy region. Then, by measuring the index of fuzziness of the subsets $B \cup\left\{x_{i}\right\}$ and $W \cup\left\{x_{i}\right\}$, the gray level is assigned to the subset with lower index of fuzziness (maximum similarity). Applying this procedure for all gray levels of the fuzzy region, we can classify them into object or background subsets. Since the method is based on measures of index of fuzziness, these measures need to be normalized by first computing the index of fuzziness of the seed subsets and calculating a normalization factor $\alpha$ according to

$$
\alpha=\frac{\psi(W)}{\psi(B)}
$$

where $\psi(W)$ and $\psi(B)$ are the IF's of the subsets $W$ and $B$, respectively. This normalization operation ensures that both initial subsets have identical index of fuzziness at the beginning of the process. It is a necessary condition since the method is based in the calculation of similarity between gray levels. Fig. 3 illustrate how the normalization works. For dark objects the method can be described as follows:

1. Compute the normalization factor $\alpha$;

2. For all gray levels $x_{i}$ in the fuzzy region compute $\psi(B \cup$ $\left.\left\{x_{i}\right\}\right)$ and $\psi\left(W \cup\left\{x_{i}\right\}\right)$;

3. If $\psi\left(W \cup\left\{x_{i}\right\}\right)$ is lower than $\alpha \psi\left(B \cup\left\{x_{i}\right\}\right)$, then $x_{i}$ is included in set $F$, otherwise $x_{i}$ is included in set $O$.

For light objects the method performs similarly except for the set inclusion in step 3. In this case, if $\psi\left(W \cup\left\{x_{i}\right\}\right)$ is lower than $\alpha \psi\left(B \cup\left\{x_{i}\right\}\right)$, then $x_{i}$ is included in set $O$, otherwise $x_{i}$ is included in set $F$.

\section{Proposed Method}

The concept presented above sounds attractive but has some limitations concerning the initialization of the seed subsets. In [5] these subsets should contain enough information about the regions and its boundaries are defined manually. The proposed method in this paper aims to overcome some of the limitations of the existing method. In fact, the initial subsets are defined automatically and they are large enough to accommodate a minimum number of pixels defined at the

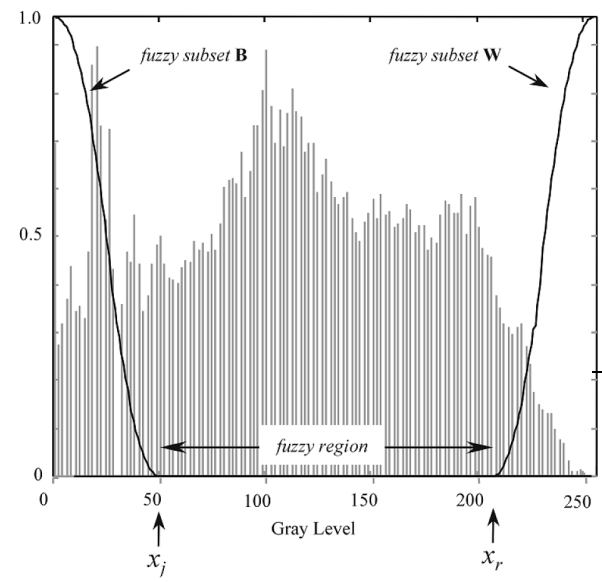

Fig. 2. Histogram and the functions for the seed subsets [5] beginning of the process. This minimum depends on the image histogram shape and it is a function of the number of pixels in the gray level intervals $[0,127]$ and $[128,255]$. It is calculated as follows

$$
\operatorname{MinPix_{Bseed}(\text {Wseed})}=P_{1} \sum_{i=0(128)}^{127(255)} h\left(x_{i}\right)
$$

where $P_{1} \in[0,1]$ and $h\left(x_{i}\right)$ denotes the number of occurrences at gray level $x_{i}$. Equation (7) can be seen as a special case of a cumulative histogram.

However, in images with low contrast the method performs poorly due to the fact that one of the initial regions contain a low number of pixels. So, previous histogram equalization is carried out in images with low contrast aiming to provide an image with significant contrast. If the number of pixels belonging to the gray level intervals $[0,127]$ or $[128,255]$ is smaller than a value $P_{M I N}$ defined by $P_{M I N}=P_{2} M N$, where $P_{2} \in[0,1]$ and $M, N$ are the dimensions of the image, the image histogram is equalized.

Equalization is carried out using the concept of cumulative distribution function [21]. The probability of occurrence of gray level $x_{i}$ in an image is approximated by

$$
p\left(x_{i}\right)=\frac{h\left(x_{i}\right)}{M N}
$$

For discrete values the cumulative distribution function is given by

$$
T\left(x_{i}\right)=\sum_{k=0}^{i} p\left(x_{k}\right)=\sum_{k=0}^{i} \frac{h\left(x_{k}\right)}{M N}
$$

Thus, a processed image is obtained by mapping each pixel with level $x_{i}$ in the input image into a corresponding pixel with level $s_{i}=T\left(x_{i}\right)$ in the output image using (9).

\section{A. Calculation of parameters $P_{1}$ and $P_{2}$}

To obtain the parameters $P_{1}$ and $P_{2}$ a statistical approach is used. Parameters $P_{1}$ and $P_{2}$ are concerned with the number of pixels of the initial intervals and histogram equalization, respectively. As the parameters are not mutually related, the statistical study is made independently.

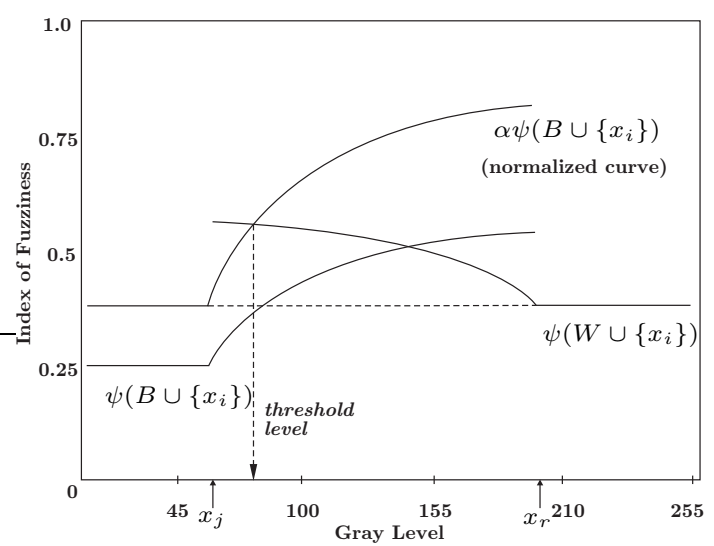

Fig. 3. Normalization step and determination of the threshold value [5] 
In this study, 30 test images are used. To determine the parameter $P_{1}$ the images in the data base presenting a significant contrast are used. Such images exhibit a significant distribution of pixels' gray levels over the interval $[0,255]$ and it is not necessary an histogram equalization. For each image, the parameter $P_{1}$ is chosen to ensure that both the IFs of the subsets $W$ and $B$ provide an increasing monotonic behavior. If $P_{1}$ is too high, the fuzzy region between the initial intervals is too small and the values of gray levels for threshold are limited. On the other hand, if $P_{1}$ is too low, the initial subsets are not representative and the method does not converge. With these minimum values of $P_{1}$ that ensure the convergence, Table I is constructed and the mean $(m)$ and the standard deviation $(\sigma)$ are calculated. After analysis of the results, the mean value of $P_{1}=39.64 \%$ is adopted.

TABLE I

\begin{tabular}{lc} 
MINIMUM VALUES OF $P_{1}(\%)$ \\
\hline Image & $P_{1}(\%)$ \\
\hline potatoes & 65 \\
shadow & 25 \\
stones & 55 \\
mouse2 & 25 \\
field & 30 \\
horses & 50 \\
statues & 35 \\
savana & 50 \\
baboon & 25 \\
boats & 40 \\
cameraman & 55 \\
lena & 35 \\
sea star & 25 \\
peppers & 40 \\
$m$ & 39.64 \\
$\sigma$ & 13.37 \\
\hline
\end{tabular}

To determine the value of $P_{2}$ the images with low contrast and parameter $P_{1}$, calculated earlier, are used. These images present a small contrast with most pixels concentrated in half side of the histogram. For these images, the minimum number of pixels in the gray level intervals $[0,127]$ or $[128,255]$ that ensures the convergence of the method is obtained by trial and error and the parameter $P_{2}$ is calculated. With these minimum values, Table II is constructed and the mean and standard deviation are also calculated. In this work, the value of $P_{2}=20 \%$ is used.

\section{EXPERIMENTAL RESULTS}

In order to illustrate the performance of the proposed methodology, 14 images are randomly selected from our original 30 images database. A manually generated groundtruth image has been defined for each image and used as a gold standard. Original images and their gold standard are illustrated in Fig. 4. Results are compared with two well established methods: the Otsu's technique (OTSU) [13] and Fuzzy C-means clustering algorithm (FCM) [6]. In this way, a comparison between fuzzy and non-fuzzy threshold algorithms
TABLE II

MinimuM VALUES OF $P_{2}(\%)$

\begin{tabular}{lc}
\hline Image & $P_{2}(\%)$ \\
\hline blocks & 17.02 \\
gearwheel & 45.99 \\
rice & 18.45 \\
zimba & 18.51 \\
mouse & 0.13 \\
blood & 35.36 \\
bird & 44.44 \\
moon & 0.51 \\
bath & 36.29 \\
mush & 20.62 \\
plane & 29.32 \\
birds & 10.92 \\
boat & 20.42 \\
airplane & 18.10 \\
ski & 11.43 \\
newspaper & 3.62 \\
$m$ & 20.70 \\
$\sigma$ & 14.30 \\
\hline
\end{tabular}

is carried out and the results of the three techniques are presented in Fig. 5. Performance is obtained by comparing the gold standard image with the corresponding image provided by the three different methods. To measure such performance, a parameter $\eta$, based on the misclassification error, has been used [17]. Thus,

$$
\eta_{(\%)}=\frac{\left|B_{O} \cap B_{T}\right|+\left|F_{O} \cap F_{T}\right|}{\left|B_{O}\right|+\left|F_{O}\right|} \times 100
$$

where $B_{O}$ and $F_{O}$ are, respectively, the background and foreground of the original (ground-truth) image, $B_{T}$ and $F_{T}$ are the background and foreground pixels in the resulting image, respectively, and $|$.$| is the cardinality of the set. This$ parameter varies from $0 \%$ for a totally wrong output image to $100 \%$ for a perfectly binary image. The performance measure for every algorithm is listed in Table III. Mean and standard deviation are also presented. The methods indicated by IM1 and IM2 represent the improved method without and with histogram equalization, respectively. After comparing results, the improved method with histogram equalization provides, in general, satisfactory results with particular attention in images with imprecise edges.

\section{CONCLUSION}

In this work, an automatic histogram threshold approach based on index of fuzziness measure is presented. This work overcome some limitations of an existing method concerning the definition of the initial seed intervals. Method convergence depends on the correct initialization of these initial intervals. After calculating the initial seeds a similarity process is started to find the threshold point. This property of similarity is obtained calculating an index of fuzziness. To measure the performance of the proposed method the misclassification error parameter is calculated. For performance evaluation purposes, 
TABLE III

PERFORMANCE OF INDIVIDUAL METHODS (\%)

\begin{tabular}{lcccc}
\hline Image & OTSU & FCM & IM1 & IM2 \\
\hline blocks & 94.38 & 80.41 & 98.87 & 99.34 \\
gearwheel & 97.85 & 97.07 & 95.59 & 95.59 \\
potatoes & 96.98 & 97.06 & 96.98 & 96.98 \\
rice & 93.51 & 85.84 & 82.06 & 95.91 \\
shadow & 90.46 & 88.30 & 93.26 & 93.26 \\
stones & 96.59 & 95.95 & 97.05 & 97.05 \\
zimba & 97.60 & 84.67 & 96.55 & 98.86 \\
mouse & 49.00 & 85.87 & 41.68 & 57.68 \\
mouse2 & 73.56 & 59.09 & 79.63 & 79.63 \\
blood & 95.61 & 95.73 & 85.09 & 85.09 \\
bird & 87.88 & 76.98 & 89.40 & 89.40 \\
moon & 26.56 & 99.97 & 99.53 & 91.40 \\
bath & 62.65 & 55.92 & 76.32 & 76.32 \\
field & 93.36 & 90.71 & 96.28 & 96.28 \\
& & & & \\
$m$ & 82.57 & 85.25 & 87.73 & 89.48 \\
$\sigma$ & 21.91 & 13.58 & 15.28 & 11.58 \\
\hline
\end{tabular}

results are compared with two well established methods: the Otsu's technique and the Fuzzy C-means clustering algorithm. After results analysis we can conclude that the proposed approach presents a higher performance for a large number of tested images.

\section{REFERENCES}

[1] W. K. Pratt, Digital Image Processing, third edition ed. John Wiley \& Sons, Inc, 2001

[2] A. S. Pednekar and I. A. Kakadiaris, "Image segmentation based on fuzzy connectedness using dynamic weights," Image Processing, IEEE Transactions on, vol. 15, no. 6, pp. 1555-1562, June 2006.

[3] S. Sahaphong and N. Hiransakolwong, "Unsupervised image segmentation using automated fuzzy c-means," Computer and Information Technology, 2007. CIT 2007. 7th IEEE International Conference on, pp. 690-694, Oct. 2007.

[4] O. J. Tobias, R. Seara, and F. A. P. Soares, "Automatic image segmentation using fuzzy sets," in Proceedings of the 38th Midwest Symposium on Circuits and Systems, vol. 2, 1996, pp. 921-924.

[5] O. J. Tobias and R. Seara, "Image segmentation by histogram thresholding using fuzzy sets," in IEEE Transactions on Image Processing, vol. 11, 2002.

[6] C. V. Jawahar, P. K. Biswas, and A. K. Ray, "Investigations on fuzzy thresholding based on fuzzy clustering," Pattern Recognition, vol. 30, no. 10 , pp. $1605-1613,1997$.

[7] K. S. Chuang, H. L. Tzeng, S. Chen, J. Wu, and T. J. Chen, "Fuzzy c-means clustering with spatial information for image segmentation," Computerized Medical Imaging and Graphics, vol. 30, no. 1, pp. 9-15, 2006.

[8] L. K. Huang and M. J. J. Wang, "Image thresholding by minimizing the measures of fuzziness," Pattern Recognition, vol. 28, no. 1, pp. 41-51, 1995.

[9] H. R. Tizhoosh, "Image thresholding using type II fuzzy sets," Pattern Recognition, vol. 38, pp. 2363-2372, 2005.

[10] A. Rosenfeld and P. de la Torre, "Histogram concavity analysis as an aid in threshold selection," $S M C$, vol. 13, no. 3, pp. 231-235, March 1983.

[11] J. S. Wezka and A. Rosenfeld, "Histogram modification for threshold selection," IEEE Transactions on Systems, Man and Cybernetics, vol. 9, pp. 38-52, 1979.

[12] T. Ridler and S. Calvard, "Picture thresholding using an iterative selection method," IEEE Transactions on Systems, Man and Cybernetics, vol. 8, no. 8, pp. 630-632, Aug. 1978.

[13] N. Otsu, "A threshold selection method from gray level histograms," IEEE Trans. Systems, Man and Cybernetics, vol. 9, pp. 62-66, 1979.
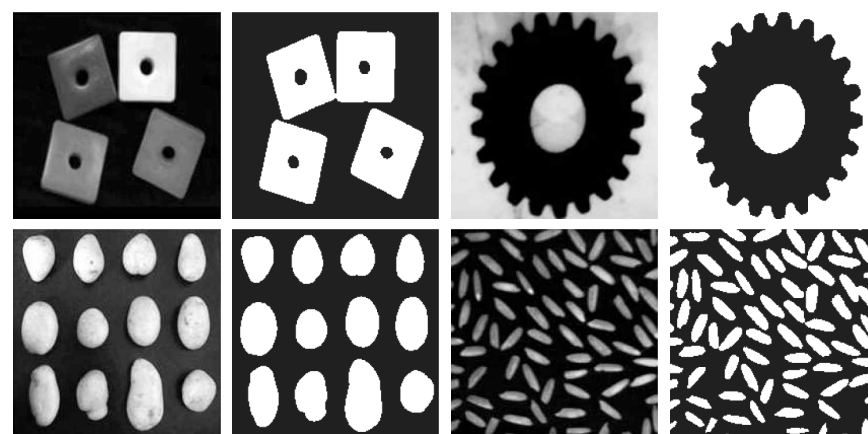

Priva

1101015 (1) 10
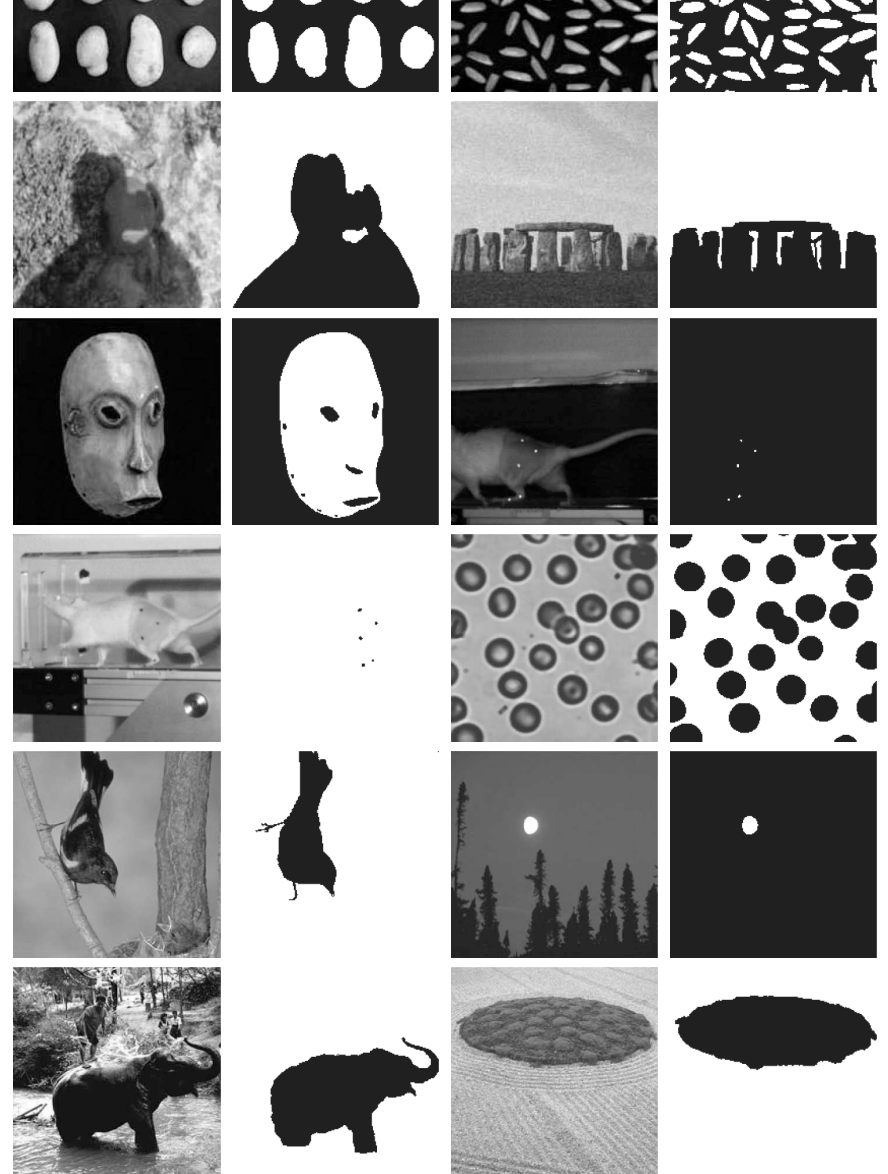

Fig. 4. Test images and the corresponding ground-truth images

[14] J. Kittler and J. Illingworth, "Minimum error thresholding," Pattern Recognition, vol. 19, no. 1, 1986

[15] J. N. Kapur, P. K. Sahoo, and A. K. C. Wong, "A new method for graylevel picture thresholding using the entropy of the histogram," Graphical Models and Image Processing, vol. 29, pp. 273-285, 1985.

[16] T. Pun, "A new method for gray-level picture thresholding using the entropy of the histogram," Signal Processing, vol. 2, no. 3, pp. 223237, 1980.

[17] M. Sezgin and B. Sankur, "Survey over image thresholding techniques and quantitative performance evaluation," Journal of Electronic Imaging, vol. 13, no. 1, pp. 146-165, January 2004.

[18] C. Murthy and S. Pal, "Fuzzy thresholding: Mathematical framework, bound functions and weighted moving average technique," Pattern Recognition Letters, vol. 11, pp. 197-206, 1990.

[19] N. R. Pal and J. C. Bezdek, "Measuring fuzzy uncertainty," in IEEE Transactions on Fuzzy Systems, vol. 2, 1994.

[20] A. Kaufmann, Introduction to the theory of fuzzy subsets. New York: Academic Press, 1975, vol. I.

[21] R. C. Gonzalez and R. E. Woods, Digital Image Processing. AddisonWesley Publishing Company, 1993. 


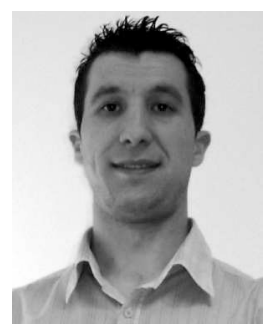

Nuno Vieira Lopes graduated in Computer and Electrical Engineering from the University of Coimbra, Portugal in 2002. Currently, he is a Ph.D. student at University of Trás-os-Montes and Alto Douro (UTAD) and he is a member of the Biosystems Engineering group at the Centre for the Research and Technology of Agro-Environment and Biological Sciences (CITAB) of UTAD since 2006. His research interests are fuzzy logic theory, digital image processing and object tracking.

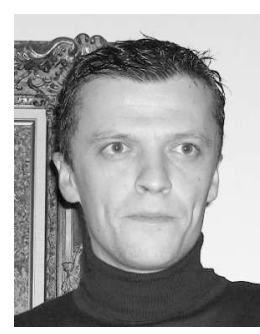

UTAD.

Pedro A. Mogadouro do Couto graduated in Electrical Engineering from the University of Trás-osMontes and Alto Douro (UTAD), Portugal in 1999. He obtained the M.Sc. degree in Engineering Technologies in 2003 and the Ph.D. degree in Electrical Engineering (Computer Vision) in 2007 from UTAD, Portugal. Currently, he is an Assistant Professor in the Engineering Department at UTAD. He is a researcher in the Biosystems Engineering group at the Centre for the Research and Technology of AgroEnvironment and Biological Sciences (CITAB) of

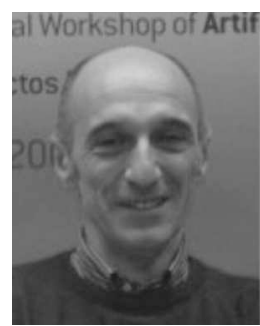

Humberto Bustince is Associate Professor at the Department of Automatics and Computation, Public University of Navarra, Spain. He holds a Ph.D. degree in Mathematics from Public University of Navarra from 1994. His research interests are fuzzy logic theory, extensions of fuzzy sets (type-2 fuzzy sets, Atanassov's intuitionistic fuzzy sets), fuzzy measures, aggregation operators and fuzzy techniques for image processing. He is author of more than 30 research peer reviewed papers.

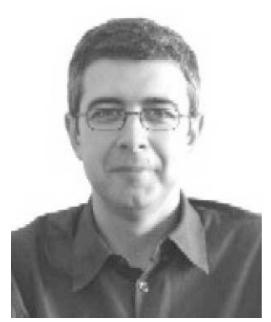

Pedro Melo-Pinto is Full Professor in the Engineering Department at UTAD. He obtained the Ph.D. degree in Applied Informatics in 2004 from the University of Trás-os-Montes e Alto Douro (UTAD), Portugal. He is currently Vice-Director and the leading researcher in the Biosystems Engineering group at the Centre for the Research and Technology of Agro-Environment and Biological Sciences (CITAB) of UTAD. His research interests are fuzzy logic and extensions of fuzzy sets, digital image processing and computer vision. 

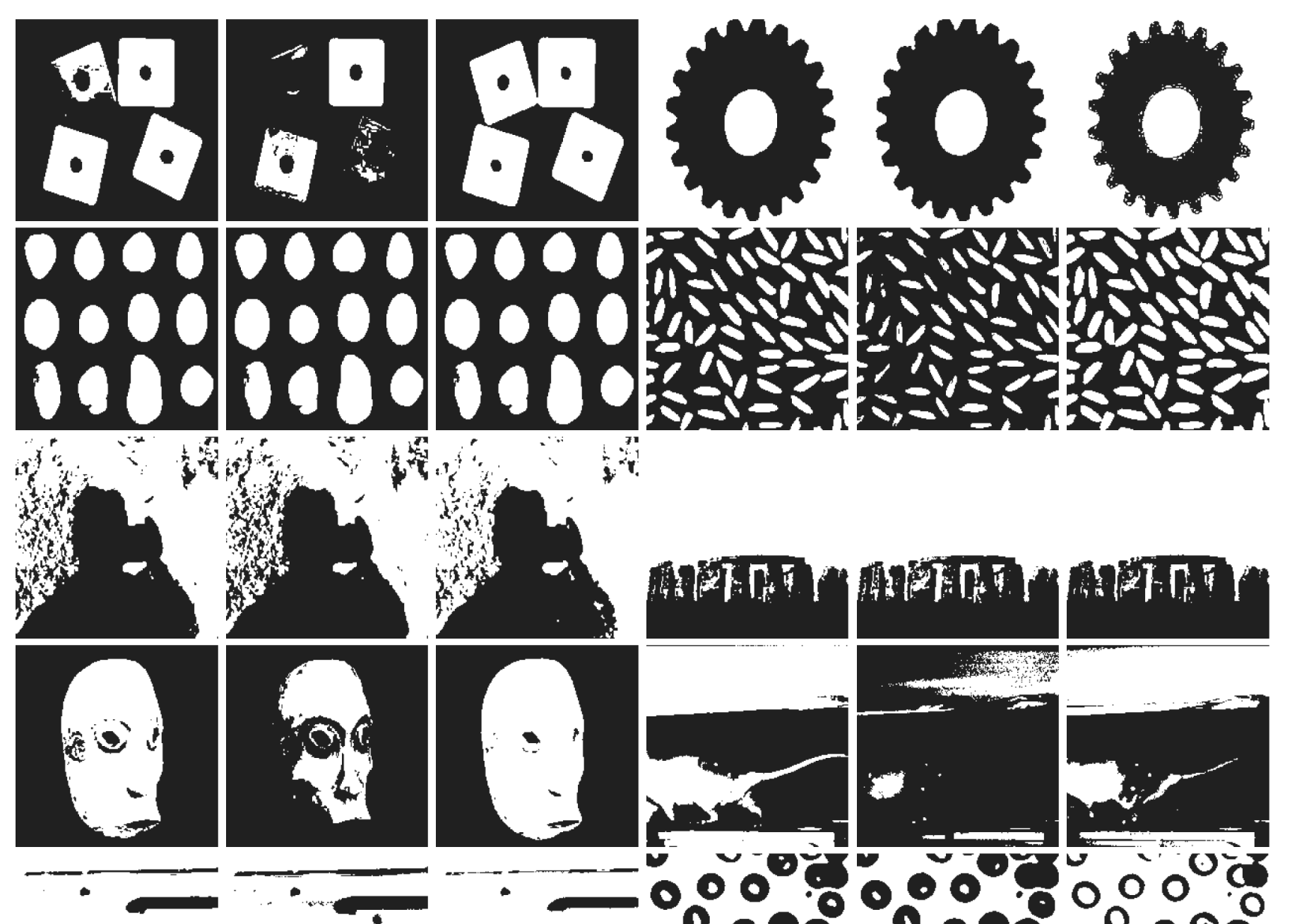

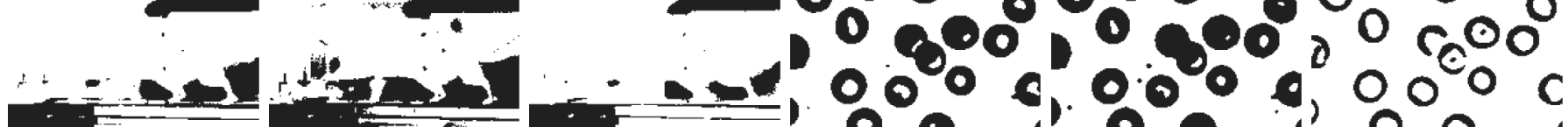
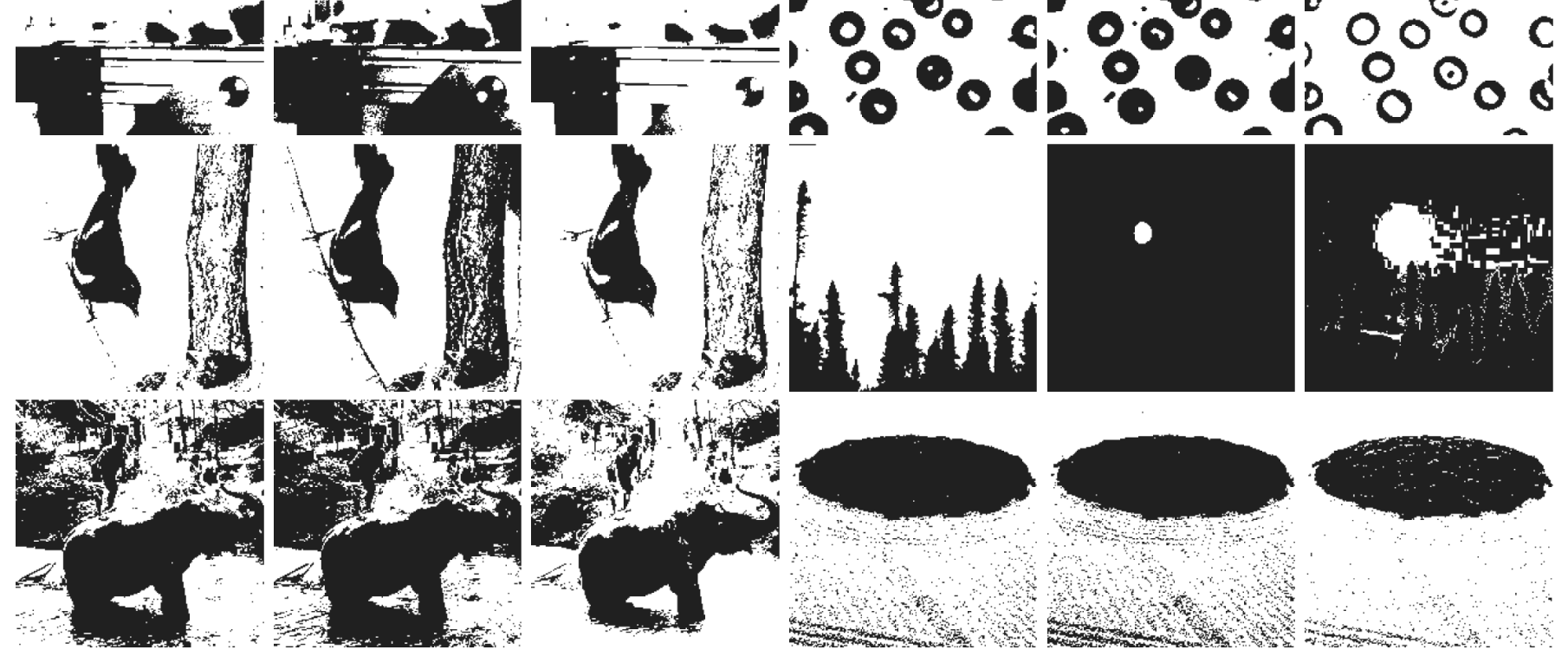

Fig. 5. Results of three algorithms. For each image, from left to right: Otsu's technique, Fuzzy C-means algorithm and final improved method 